\title{
Best Practices for Selection of Excipients for Paediatrics - Workshop Reflection
}

\author{
Authors \\ Smita Salunke ${ }^{a *}$, David Clapham ${ }^{b}$, Anjali Agrawal $^{c}$, Kevin Hughes ${ }^{d}$, Tony Nunn ${ }^{\dagger}$ \\ a European Paediatric Formulation Initiative (EuPFI), University College London School of Pharmacy, \\ London, UK; s.salunke@ucl.ac.uk \\ b Independent Pharmaceutical Consultant, 14 Tailors, Bishops Stortford,CM23 4FQ, UK, \\ david.clapham@ntlworld.com \\ cBristol Myers Squibb, 181 Passaic Avenue, Summit, NJ; anjali 50@yahoo.com \\ d IPEC (International Pharmaceutical Excipients Council) and Colorcon Ltd, Dartford UK \\ ‘ Department of Women's and Children's Health, University of Liverpool, Liverpool Women's Hospital, \\ Liverpool, L8 7SS, UK. a.j.nunn@liverpool.ac.uk \\ On behalf of European Paediatric Formulation Initiative \\ *Corresponding author: s.salunke@ucl.ac.uk
}

\section{Conflict of interest}

Smita Salunke, David Clapham, Kevin Hughes and Tony Nunn are members of the European

Paediatric Formulation Initiative.

Anjali Agrawal is a member of the International Consortium for Innovation and Quality in

Pharmaceutical Development (IQ Consortium)

This Workshop did not receive any specific grant from funding agencies in the public, commercial, or not-for-profit sectors. None of the authors or speakers received any compensation or funding for the Workshop or manuscript publication.

\section{Keywords}

- $\quad$ Drug development

- Pharmaceutical

- Excipients

- Risk assessment

- Medicines

- Paediatric
- Formulation

- EuPFI

- Workshop

- Choice 


\section{Abstract}

2 The development of age appropriate formulations for the paediatric population has become

3 one of the key areas of focus for the pharmaceutical industry - with a subsequent influence

4 on excipient use. Selection of excipients with appropriate safety and tolerability is a major

5 hurdle in paediatric formulation development. Various factors influence selection of

6 excipients, including target age group, route of administration, dosage form. Evaluation of

7 these factors and a clear rationale and justification is expected by the regulators when it

8 comes to selecting excipients for paediatric formulation. Scientists are encouraged to apply

9 the principle of benefit to risk balance to assess the suitability of excipients to the specific

10 paediatric population for whom the formulation is intended. In order to understand how

11 scientists, approach the task of establishing the risk to benefit analysis, a workshop was organised by the European Paediatric Formulation Initiative (EuPFI) to reflect on the current

13 scenario and the different practices employed by formulation scientists in the selection of

14 excipients for paediatric formulations. Aspects assessed by regulators were also

15 canvassed. Finally, the participants were asked to comment on how selecting excipients for use in paediatric formulations may differ from the considerations applied in selecting excipients for formulations for other age groups. Based on the workshop discussion, some recommendations and questions to consider emerged regarding the selection of excipients in paediatric drug development. These best practice recommendations provided a good starting point for a more systematic strategy for selecting excipients for paediatric

21 formulation development. 


\section{Introduction}

30 Recent regulatory directives have put the development of paediatric medicines into the spotlight. The development of age appropriate formulations for the paediatric population has become one of the key areas of focus for the pharmaceutical industry - with a subsequent influence on excipient use. Along with the other technical and quality related requirements of formulations, swallowability and palatability are the key attributes for patient and carer acceptability of oral dosage forms for children 1 . Excipients offer benefits in these areas as useful aids to formulation scientists. However, finding or selecting excipients with appropriate safety and tolerability is a major hurdle in paediatric formulation development. The immaturity of organs, particularly of very young children, means that certain excipients (e.g., propylene glycol, ethanol) cannot be metabolized in the same way as an adult and can lead to deleterious adverse effects ${ }^{2,3,4}$. So, it is important to look at the absorption of these materials, how they are metabolised, and is there any potential for them to accumulate and cause toxic effects. A number of excipients with attributes well matched to paediatric formulations are available; for example, fillers and disintegrants that provide good texture (mouth feel) for orally dispersible tablets and coating materials that prevent premature release of the drug in saliva (for taste-masking). Texture is also important for other oral dosage forms such as viscous solutions, emulsions, and suspensions ${ }^{5}$. However, a formulation scientist needs to have a thorough understanding of the attributes (physicochemical and safety) of excipients used for a given type of formulation, and when certain materials should be used in preference to others. In all regulatory submissions, the reviewers expect a clear rationale for the selection of excipients, including the role of the excipient and amounts used 6 . In general, the selection of excipients for a particular formulation should be based on the experimental evaluation of a range of candidates and their exposure to the child. The best science and clinical practice must be applied in selection of excipients for children. There is an impressive wealth of knowledge and know-how in terms of applying good scientific common sense in selecting the most appropriate excipients for formulations for adults within the industry. However, no general well-defined principles or best practices exist for selection of the most appropriate excipient for paediatric formulation development. Commonly, these decisions are based on "institutional preconceptions" or personal experience. The European Medicines Agency (EMA|) guidance document and expert opinion advocates a risk-based approach for selection of excipients but exactly "how" this is to be conducted is not specified ${ }^{6,7}$. The diversity of strategies currently 
employed can lead to variety of practices ${ }^{7,8}$. Several additional challenges remain when applying risk assessment in selection of excipients for neonates, children and adolescents. These include, but are not limited to, data and knowledge gaps; methodological limitations; difficulties in aggregating/comparing risks and benefits and in combining human (adult) data with data extrapolated from animal studies; lack of harmonization of concepts; and complexities in communicating best practices. To understand the different practices used by formulation scientists and the aspects assessed by the regulators in selection of excipients for paediatrics, the EuPFI Excipients workstream members constructed and organised a workshop on "Best Practices for Selection of Excipients for Paediatrics". The workshop was undertaken as a half day preconference workshop to the $10^{\text {th }}$ annual conference of EuPFI held in London on $11^{\text {th }}$ September $2018^{9}$. The key objectives of the workshop were

1. To foster discussion among various stakeholders involved in development of medicines for children on understanding current practices in selecting excipients for development of paediatric formulations

2. To identify the questions or elements to be considered in the process of selection of excipients and how selection process may change and evolve during the product development process.

The purpose of this report is to summarise the outcomes of scenarios and tasks given to participants and discussion statements that participants contributed to an open floor discussion. While the improvement of the practice for selection of excipients for paediatric formulation development will require the involvement of broadest possible spectrum of disciplines such as excipients suppliers, manufacturers, regulators, industry representatives, this workshop report is none-the-less, an important first step towards further research and dialogue between key stakeholders. It offers formulation scientists and researchers involved in the development of medicines for children, recommendations and questions to consider whilst selecting excipients for paediatric formulations.

\section{Methods}

86 A scenario workshop was organised to gather knowledge about participants understanding and experience with selection of excipients for paediatric formulation development, by looking into their opinion and feedback towards defined scenarios (Figure 1). The scenario workshop is a participatory 
method that involves groups of participants interacting with other participants to exchange knowledge, experience, develop common vision, debate, provide criticism and produce a plan of action for potential future developments ${ }^{10}$. Following an introduction to the field of excipients, legislation concerning the use of excipients in paediatric products and the structure of the workshop, participants were distributed into small groups. This subdivision was necessary to balance the various interests of the different roles within the groups (e.g., regulatory, industry, academic) and to include them on an equal basis. The groups were chosen to, as far as possible, include participants who had some experience in the use and choice of excipients in product development and those who were less

97 familiar with the topic.

The participants responded to up to two out of three scenarios posed to them concerning

99 - the use of a novel excipient

$100 \quad$ the use of an established excipient

101 the choice between a range of possible suspending agents

102 The scenarios included a hypothetical but realistic formulation challenge and participants were asked

103 - To apply their current selection practice to the scenario provided and suggest if the excipient is suitable for the formulation in development and justify the selection.

- To consider what questions they would need to ask themselves in deciding whether or not to use the specific potential excipient in the formulation.

109 Each group was supported by a facilitator from the EuPFI excipients workstream to provide guidance

110 when needed and to answer any questions posed to them based on a pre agreed facilitator brief.

111 Within the groups, the workshop facilitators asked all participants to discuss and deliberate on their

112 delegated tasks and then present their conclusions to all workshop participants. They were also

113 asked to provide critical comment on the feedback provided by the other 3 breakout discussion

114 groups. Facilitators were encouraged to stimulate discussion and help the group reach its own

115 conclusions and to avoid instructing the group as far as possible. The four discussion groups worked

116 towards developing a precise list of questions or elements that they considered during the selection of

117 excipients as per their scenario and an elected representative member provided a summary of the

118 outcome of their discussions for comment by others groups as a whole. After the four small groups 
119 presented their feedback, a common list of questions and elements was created and compared with

120 the list of questions and elements created by the workshop organisers and facilitators for each

121 scenario prior to the workshop. As an example, the list of questions and elements created for the 122 scenario on use of an established excipient is presented in Table 1. The list of questions developed in

123 advance by facilitators was presented at the end of the workshop after receiving the feedback from 124 participants. The feedback and additional questions identified by the participants was collated by the 125 workshop facilitators and is summarised below in results and discussion section.

\section{Results and Discussion}

128 The workshop brought together stakeholders involved in paediatric drug development for intensive 129 and structured discussions on selection of excipients for paediatric formulations. It was attended by

13057 participants including representatives from academia, industry, regulators, hospitals and other 131 organisations (Figure 2).

132 The purpose was not to make a final declaration about the directions that should be taken, but to

133 further the examination of practices in selection of excipients in the open networked environments, based on the scenarios given to participants. Gratifyingly, all the groups found the exercise helpful

135 and identified that the decision about whether or not to use a particular excipient is multifactorial.

136 Many groups structured their responses under broad headings including such considerations as

137 technical aspects, safety, acceptability, biopharmaceutical aspects, manufacturability, cost,

138 commercial considerations and information that they felt that they could or should supply to enhance

139 discussions with excipient suppliers.

140 Although structured in different ways all groups came to a high level of agreement on the factors to 141 consider. In cases, where a point was mentioned by only one or two of the groups the other groups 142 agreed that the point was valid and should have been included in their own analysis. There was also 143 general agreement regarding what would be different when considering an excipient for a paediatric 144 formulation versus one for an adult population and what additional factors should be considered for 145 using a novel excipient versus an established one (Figure 3).

146 The outcome was in good agreement with a set of elements and questions that the workshop 147 facilitators had developed prior to the event. For example, the questions collated from the workshop 148 participant output and those previously identified by the facilitators for a hypothetical established 
149 suspending agent called SuPlus derived from a natural food source for use in a liquid dosage form

150 are presented in Table 1 and Table 2 respectively

151 The major question that the participants failed to ask was 'Do I actually need that particular excipient

152 at all? Participants agreed that in fact the majority of factors that need to be considered are the same

153 no matter what the target age group. The main difference is in the level of toxicological information

154 required - particularly whether age appropriate studies had been conducted or not. The other main

155 differences were a focus on using the minimum number and lowest possible use level to achieve the

156 required technical effect and an increased clarity on justifying the need for the excipient.

157 For a scenario on potential novel excipient, the questions were similar but there was an increased 158 focus on

159 - The level and type of toxicity data that is available

- Robustness of the evidence base for the proposed technical and/or clinical benefit of using the novel excipient as opposed to a more established one.

- The reliability and reproducibility of supply

163 There is also a need to understand the funding model of the excipient supplier, for example, will they

164 demand a royalty and can the product sustain that cost?

165 Overall, after the input from all the groups, a list of the common additional questions identified by

166 workshop participants was created and is presented in Table 3 below.

168 In summary, while issues of best practice for selection of excipients for paediatric formulation are still

169 being discussed, participants also stressed that a standardised risk assessment approach to aid

170 selection of excipients for paediatric products is needed. In the past, the selection of excipients during

171 formulation development may have been limited to preliminary studies like compatibility of excipient

172 with drug, drug solubility in excipient and the effect of the excipient on desired drug release. However,

173 now, a scientifically sound risk assessment and consequent appropriate use of excipients is the basis

174 for any risk reduction measures and ultimately would provide a basis for the sustainable use of

175 excipients in paediatrics. The workshop highlighted that using a scientifically valid, question-based

176 approach to excipient selection will allow formulators to optimally use those excipients to overcome

177 challenges such as poor organoleptic acceptability, non-optimal bioavailability and stability challenges

178 while optimizing the manufacturability using the manufacturing process that is most effective and 
efficient. The EuPFI excipients workstream has consider two possible themes for its future work

180 programme as an action plan from this workshop. First, the group in collaboration with Innovative

181 Quality Pediatric Working group (IQPedWG) could develop "a structured benefit risk assessment

182 framework focusing on guidelines and elements to consider during the selection and overall risk

183 assessment of excipients likely to be used in paediatric formulations". Second, the group would

184 explore the development of a risk assessment tool to systematically document the analysis for a

185 particular excipient or between multiple excipient options with similar functionality to enable decision-

186 making using the risk-benefit framework principles.

187

\section{Conclusion}

189 The workshop was successful in helping the participants to identify a reasonably comprehensive set

190 of questions that formulators should ask themselves when considering whether or not to use a

191 particular excipient in a paediatric formulation. The collated list of questions identified by the

192 participants and facilitators showed that the choice of whether or not to use an excipient and which

193 excipient to choose from a set of possible choices is a complex and multifactorial one. Participants

194 agreed that it is important that excipient choice both in terms of identity and usage level is a

195 conscious decision taking into account all relevant factors and not one made without due thought and

196 attention.

Reference:

200 1. Ternik R, Liu F, Bartlett JA, et al. Assessment of swallowability and palatability of

201 oral dosage forms in children: Report from an M-CERSI pediatric formulation workshop. Int

202 J Pharm 2018;536:570-81.

203 2. Ernest TB, Elder DP, Martini LG, Roberts M, Ford JL. Developing paediatric

204 medicines: identifying the needs and recognizing the challenges. J Pharm Pharmacol 205 2007;59:1043-55.

206 3. Al-khattawi A MA. Excipients in medicines for children: scientific and regulatory

207 paradigms. European Pharmaceutical Review

208 2014; https://www.europeanpharmaceuticalreview.com/article/24946/excipients-medicines-

209 children-scientific-regulatory-paradigms/ [accessed 16 November 2020].

210 4. Valeur KS, Holst H, Allegaert K. Excipients in Neonatal Medicinal Products: Never

211 Prescribed, Commonly Administered. Pharmaceut Med 2018;32:251-8.

212 5. Mistry P, Batchelor H. Evidence of acceptability of oral paediatric medicines: a

213 review. J Pharm Pharmacol 2017;69:361-76. 
214 6. EMA. European Medicines Agency Committee for Medicinal Products for Human

215 Use and Paediatric Committee (2013) Guideline on the pharmaceutical development of

216 medicines for paediatric use. EMA/CHMP/QWP/805880/2012 Rev. 2.

217 https://www.ema.europa.eu/en/documents/scientific-guideline/guideline-pharmaceutical-

218 development-medicines-paediatric-use en.pdf [accessed 16/11/20]. 2013.

$2197 . \quad$ Turner MA, Duncan JC, Shah U, et al. Risk assessment of neonatal excipient

220 exposure: Lessons from food safety and other areas. Advanced Drug Delivery Reviews

$221 \quad 2014 ; 73: 89-101$.

222 8. IPEC. The International Pharmaceutical Excipients Council, Qualification of

223 Excipients for Use in Pharmaceuticals.

224 https://ipecamericas.org/sites/default/files/ExcipientQualificationGuide.pdf [Accessed

225 24/11/2020]. 2008.

226 9. Clapham D, Hughes K, Anjali A, Nunn A, Salunke S. EuPFI 10th Conference -

227 workshop presentation. Best practices in the selection of excipients for paediatrics.

228 http://www.eupfi.org/past-conferences/10th-eupfi-conference/. 2018.

229 10. Slocum N. Participatory Methods Toolkit: a Practitioner's Manual: King Baudouin

230 Foundation. https://cris.unu.edu/sites/cris.unu.edu/files/Toolkit.pdf [Accessed 24/11/2020];

2312003. 